\title{
Haemodynamics imaging of swine segmental kidney artery using duplex Doppler technique
}

\author{
Roman Aleksiewicz ${ }^{1}$, Krzysztof Lutnicki², Marcin Bojarski ${ }^{3}$, \\ Ilona Al-Mutari ${ }^{4}$, Aneta Bocheńska ${ }^{3}$ \\ ${ }^{1}$ Unit of Animal Surgery and Diagnostic Imaging, Poznan University of Life Sciences, 60-637 Poznań, Poland \\ ${ }^{2}$ Department and Clinic of Animal Internal Diseases, Faculty of Veterinary Medicine, \\ University of Life Sciences, 20-950 Lublin, Poland \\ ${ }^{3}$ Silesian Veterinary Polyclinic Association, 41-500 Chorzów, Poland \\ ${ }^{4}$ University Children's Hospital of Cracow, 30-663 Kraków, Poland \\ provet1@poczta.onet.pl
}

Received: February 5, 2019 Accepted: June 3, 2019

\begin{abstract}
Introduction: The aim of the study was to assess the usefulness of duplex Doppler to objectify swine renal arterial flow in physiological conditions. The pig kidney was selected for its morphological similarities to the human and for the results therefore offering data to wider research. Material and Methods: Six White Large x Landrace sows, of 48.5-53 $\mathrm{kg}$ b.w. were used. Vascular flow parameters were acquired with a convex probe USG device with a duplex Doppler function using pulsed waves (frequency range of 5-7.5 MHz). Segmental kidney arterial flow was measured. Results: The RI values were within the $0.57(\mathrm{~min})$ to $0.6(\max )$ range, $\bar{x}$ RI was $0.58( \pm 0.014)$, and the $\mathrm{SD}^{2}$ value was 0.0002 . The PI index values ranged from $1.21(\mathrm{~min})$ to $1.3(\max )$, and $\bar{x}$ PI was $1.24( \pm 0.035)$. The value of $\mathrm{SD}^{2}$ was 0.00123 . In the $\mathrm{S} / \mathrm{D}$ index, the results fell between $2.2(\mathrm{~min})$ and $2.49(\mathrm{max})$, with $\bar{x}$ s/D of $2.29( \pm 0.117)$. The value of variance $\mathrm{SD}^{2}$ was 0.0139 . A double analysis of correlation between indices showed this to be almost certain and highly positive as confirmed by high correlation coefficients: $r$ RI \& PI 0.857 , $r h o$ RI \& PI $0.739, r$ RI \& S/D 0.930, rho RI \& S/D 0.941, $r$ PI \& S/D 0.913, and rho PI \& S/D 0.754. The segmental kidney arterial flow spectrum evinced falls in PSV and PSV/LDV ratio, also noticed in the filling of the spectral window, comparing the renal to an interlobar artery. Conclusion: Swine were selected because of renal anatomical and haemodynamic similarity to humans. The most relevant values and indices approximated those in humans. The study anaesthetic protocol had a minor influence on the average RI, PI, and S/D indices.
\end{abstract}

Keywords: swine, kidney, resistance index, pulsatility index, systolic/diastolic index.

\section{Introduction}

Pigs, which offer a conveniently large anatomy and relatively large body fluid and tissue resources, are being increasingly adopted as a model in scientific research $(5,15,25)$. For research purposes, farm pigs of various breeds are used, including Landrace, Yorkshire, Duroc, Hampshire, and miniature and hybrid. This is due to their morphological and functional similarities to the human body (28). The pig is a leading animal species in xenotransplantation, where ultrasound monitoring has a key application. These animals are used in research towards the standardisation of physiological parameters, including studies on the behaviour of haemodynamic parameters using the Doppler technique. This type of research finds particular application in the field of functional kidney vascularisation. The basis of porcine kidney vasculature are the renal artery, which is a vessel outside the organs, and segmental and interlobular arteries that are interstitial vessels of the kidney. The mechanism of renal autoregulation of blood flow functions by changes in the resistance (diameter) of arterioles supplying blood is autonomous and is also preserved in an isolated kidney $(8,29,31)$. The precapillary vessels are of crucial importance for 
ensuring the flow stability; the smooth sphincter muscles are strongly influenced by xenobiotics, humoral factors, and cellular metabolites. Systemic inflammatory response syndrome (SIRS), poisoning, and burns promote the development of oedema which mechanically reduce the light of arterioles and arteries within an organ (32). The use of real-time two-dimensional ultrasound, including Doppler examination, allows the position of the measured volume to be visualised on the background of the anatomical image of an organ, to measure flows in the selected vessel, and to precisely calculate the flow rate $(12,32)$. Haemodynamic indicators, such as the resistance index (RI, also known as the Pourcelot index) describing the relationship between blood pressure and blood flow, pulsatility index (PI, also known as Gosling's index) indicating pulsatile nature of blood flow, and S/D index (systolic/diastolic index) $(17,18,28)$ can also be provided by such examinations. The flow spectrum in the segmental artery under physiological conditions is characterised by a decrease in the ratio of the systolic velocity (PSV) to the latediastolic velocity (LDV) (flattening of the graph), spectral window filling, and decrease in PSV values compared to the renal and interlobar arteries $(17,27$, 28). Thus, early disturbances of blood flow in the arterial vascular system can be noted in the area of arteries within an organ by duplex Doppler USG techniques.

The study aimed to determine the value of flows under physiological conditions in the field of segmental arteries in pigs by applying the technique. There are few reports in the available literature on the physiological parameters of blood flows regarding this area of vascularisation in the domestic pig. The research carried out on this species was aimed at providing the morphometric data that could be used in continued research works. The selection of the species was made taking into account the morphological and functional similarities of the pig to human kidneys (30), and the possibilities of using the results for further research on human diseases designed using the pig model.

\section{Material and Methods}

Six White Large $\mathrm{x}$ Landrace breed sows, weighing 48.5-53 kg were used. One week of acclimatisation before the start of the test was specified. The animals were kept at $18^{\circ} \mathrm{C}$ with constant access to feed and water. The sows were fasted for $24 \mathrm{~h}$ and were deprived of water for an hour before the experiment. Half an hour before the test, premedication was administered. Atropinum sulfuricum at a dose of $0.04 \mathrm{mg} / \mathrm{kg}$ s.c., azaperone at a dose of $3 \mathrm{mg} / \mathrm{kg}$ i.m., and ketamine at a dose of $10 \mathrm{mg} / \mathrm{kg}$ i.m. were used. Subsequently, infusion anaesthesia was implemented: propofol at 4-7 $\mathrm{mg} / \mathrm{kg} / \mathrm{h}$ and midazolam at $0.1-$
$0.5 \mathrm{mg} / \mathrm{kg} / \mathrm{h}$. The examination of vascular flow parameters was carried out using the PICO USG device from Samsung Medison (Korea) with the duplex Doppler function based on the pulsed-wave Doppler (PW Doppler) method, with a convex probe (frequency range of 5-7.5 MHz). The measurements concerned the flow in segmental kidney arteries (Figs 1 and 2). The ultrasound examination consisted of imaging the left kidney in the B-mode, using the colour Doppler function and assessing the flow with the spectral Doppler. The examination was performed using B-mode. The assumed test depth was $10 \mathrm{~cm}$, the probe frequency $5 \mathrm{MHz}$, and dynamic range $95 \mathrm{~dB}$. The default gain was set and was correlated with the determined test depth. The study protocol in B-mode included the assessment of the kidney structure during the first measurement: size in the longitudinal and transversal dimensions, echogenicity of the cortex, cortico-spinal ratio, outline of the capsule, and evaluation of renal pelvic echoes. A colour Doppler function was used for imaging blood flow and colourcoding flow direction in real time to see the intrarenal arteries. The gate width was from 2 to $4 \mathrm{~mm}$ depending on the diameter of the vessel, and the angle of insonation between the ultrasound beam and the vector of blood flow direction in the Doppler measurement did not exceed 60 degrees. The maximum and minimum flow rates were determined. Images in freeze mode were recorded in the device's memory for additional evaluation of spectral parameters. The haemodynamic status of segmental kidney arteries was measured in real time with simultaneous imaging in B-mode and PW Doppler. Haemodynamic index values were determined using the software of the device and were RI, PI, and S/D. Five measurement periods were established at 15-min intervals. Ten measurements were taken during each period. The test results are tabulated as mean values (Tables 1, 2, and 3) and are presented in Figs 1 and 2. Statistical analysis of results and graphic illustrations were made using Statistica v.10.1 software (StatSoft, now Tibco, USA).The statistical average $(\bar{x})$ and standard deviation (SD), minimum ( $\min )$, maximum $(\max )$, and the value of variance $\left(\mathrm{SD}^{2}\right)$ were calculated for all results. The Pearson correlation coefficient $r$ and the rho coefficient of the Spearman correlation between the values of individual indices (RI and PI, RI and S/D, and PI and S/D) were also found. Selected indicator result correlations were calculated at $\mathrm{P} \leq 0.05$. The results and their interpretations are presented in the form of diagrams and tables.

\section{Results}

Records of the flow spectrum in segmental kidney arteries were made (Figs 1 and 2).

A constant one-way blood flow was seen in the diastolic phase during Doppler examination in the 
swine segmental artery of the kidney. The difference between the maximum and minimum velocity and decrease in diastolic flow associated with peripheral vascular resistance is also shown (Fig. 1). Parameters for systolic (PSV) and late-diastolic (LDV) rates of blood flow were determined (Table 1).

The determined values of Pearson's (r) and Spearman's (rho) correlation coefficients are shown in Table 3.

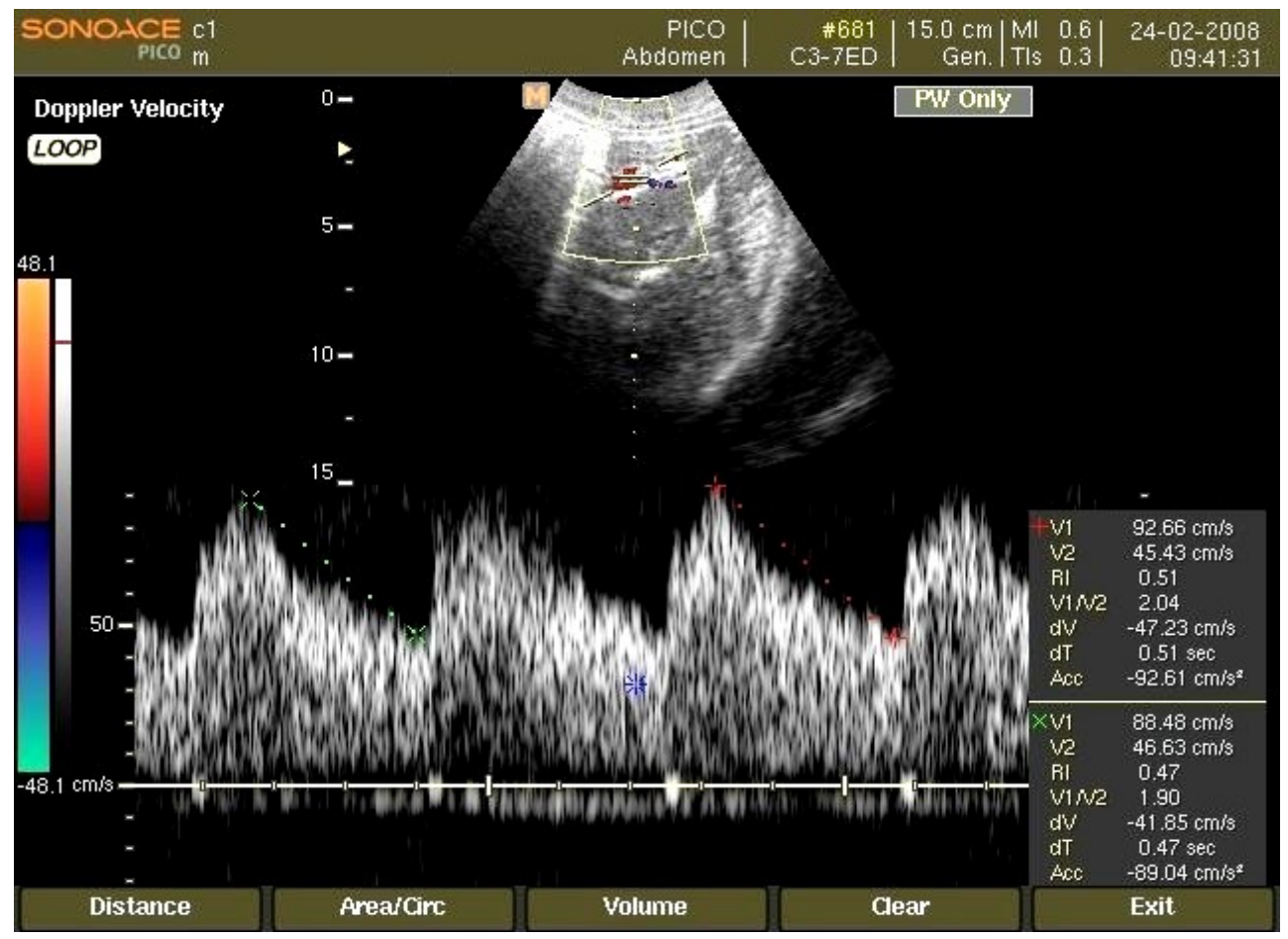

Fig. 1. Blood flow spectrum recorded in the segmental artery of the swine kidney during physiological conditions. $\mathrm{V}_{1}$ - maximum of peak systolic velocity (PSV), $\mathrm{V}_{2}$ - minimum end diastolic velocity (ESV), $\mathrm{V}_{1} / \mathrm{V}_{2}$ - the ratio of the maximum to the minimum velocity, $d v$ - difference between the maximum and minimum velocity, $\mathrm{dt}$ - observation time, Acc - value of acceleration/delay (on the graph: the angle of the curve's rise - positive value; the angle of the curve's fall- negative value)

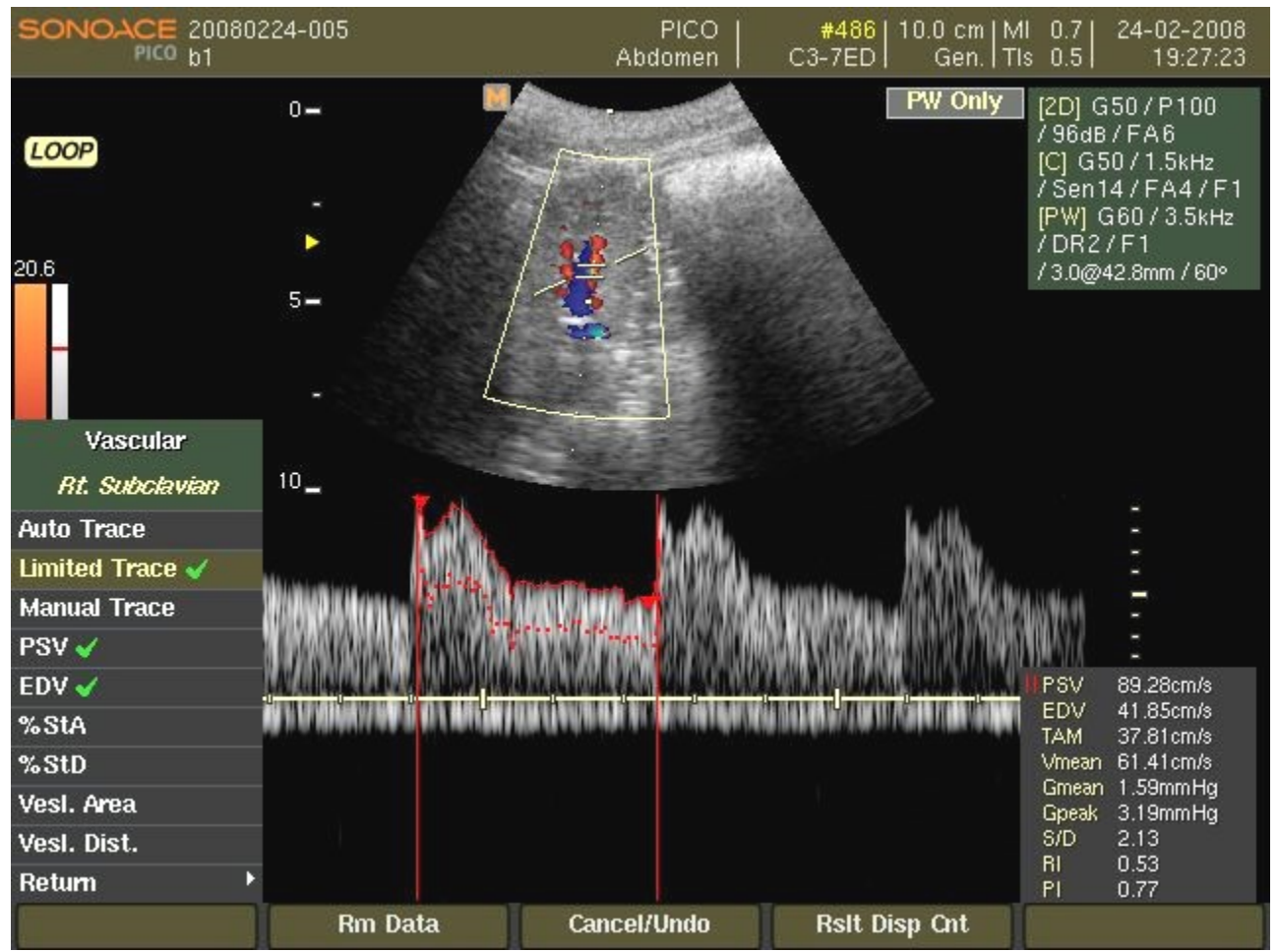

Fig. 2. Blood flow spectrum recorded in the segmental artery of the swine kidney during physiological conditions 
Table 1. Values of flows in the segmental kidney artery

\begin{tabular}{|c|c|c|c|c|}
\hline Parameter & $\bar{x}(\mathrm{SD})$ & Min & Max & Time (min) \\
\hline $\mathrm{V} 1(\mathrm{~cm} / \mathrm{s})$ & $92.66( \pm 0.95)$ & 91.45 & 93.89 & \\
\hline $\mathrm{V} 2(\mathrm{~cm} / \mathrm{s})$ & $46.43( \pm 0.88)$ & 45.05 & 47.07 & \\
\hline $\mathrm{V} 1 / \mathrm{V} 2$ & $2.04( \pm 0.16)$ & 1.98 & 2.21 & 0 \\
\hline $\operatorname{dv}(\mathrm{cm} / \mathrm{s})$ & $-43.23( \pm 0.15)$ & -44.25 & -42.16 & \\
\hline $\operatorname{Acc}\left(\mathrm{cm} / \mathrm{s}^{2}\right)$ & $-88.61( \pm 0.18)$ & -90.12 & -87.32 & \\
\hline $\mathrm{V} 1(\mathrm{~cm} / \mathrm{s})$ & $88.26( \pm 0.75)$ & 87.02 & 89.12 & \\
\hline $\mathrm{V} 2(\mathrm{~cm} / \mathrm{s})$ & $46.05( \pm 0.65)$ & 45.65 & 47.23 & \\
\hline $\mathrm{V} 1 / \mathrm{V} 2$ & $1.89( \pm 0.26)$ & 1.77 & 1.98 & 15 \\
\hline $\mathrm{dv}(\mathrm{cm} / \mathrm{s})$ & $-42.45( \pm 0.16)$ & -43.26 & -41.24 & \\
\hline $\operatorname{Acc}\left(\mathrm{cm} / \mathrm{s}^{2}\right)$ & $-71.95( \pm 0.22)$ & -72.25 & -70.65 & \\
\hline $\mathrm{V} 1(\mathrm{~cm} / \mathrm{s})$ & $89.08( \pm 0.62)$ & 88.83 & 89.87 & \\
\hline $\mathrm{V} 2(\mathrm{~cm} / \mathrm{s})$ & $47.83( \pm 0.73)$ & 46.66 & 49.01 & \\
\hline $\mathrm{V} 1 / \mathrm{V} 2$ & $1.86( \pm 0.19)$ & 1.65 & 1.98 & 30 \\
\hline $\mathrm{dv}(\mathrm{cm} / \mathrm{s})$ & $-41.25( \pm 0.18)$ & -42.25 & -40.25 & \\
\hline $\operatorname{Acc}\left(\mathrm{cm} / \mathrm{s}^{2}\right)$ & $-65.48( \pm 0.18)$ & -67.55 & -64.15 & \\
\hline $\mathrm{V} 1(\mathrm{~cm} / \mathrm{s})$ & $86.09( \pm 0.83)$ & 85.14 & 86.77 & \\
\hline $\mathrm{V} 2(\mathrm{~cm} / \mathrm{s})$ & $46.63( \pm 0.77)$ & 46.25 & 47.88 & \\
\hline $\mathrm{V} 1 / \mathrm{V} 2$ & $1.85( \pm 0.22)$ & 1.76 & 1.92 & 45 \\
\hline $\mathrm{dv}(\mathrm{cm} / \mathrm{s})$ & $-39.46( \pm 0.11)$ & -40.45 & -38.24 & \\
\hline $\operatorname{Acc}\left(\mathrm{cm} / \mathrm{s}^{2}\right)$ & $-68.03( \pm 0.22)$ & -70.05 & -66.93 & \\
\hline $\mathrm{V} 1(\mathrm{~cm} / \mathrm{s})$ & $87.27( \pm 0.64)$ & 86.45 & 87.32 & \\
\hline $\mathrm{V} 2(\mathrm{~cm} / \mathrm{s})$ & $47.24( \pm 0.65)$ & 46.76 & 47.44 & \\
\hline $\mathrm{V} 1 / \mathrm{V} 2$ & $1.85( \pm 0.23)$ & 1.77 & 1.94 & 60 \\
\hline $\mathrm{dv}(\mathrm{cm} / \mathrm{s})$ & $-41.32( \pm 0.17)$ & -42.15 & -40.38 & \\
\hline $\operatorname{Acc}\left(\mathrm{cm} / \mathrm{s}^{2}\right)$ & $-73.32( \pm 0.15)$ & -75.25 & -72.33 & \\
\hline
\end{tabular}

$V_{1}$ - maximum of peak systolic velocity, $V_{2}$ - minimum end diastolic velocity, $V_{1} / V_{2}-$ the ratio of the maximum to the minimum velocity, $\mathrm{dv}$ - difference between the maximum and minimum velocity, Acc - value of acceleration/delay

Table 2. Values determined for RI, PI, and S/D indices

\begin{tabular}{llllllllll}
\hline Index & Value (SD) & Value (SD) & Value (SD) & Value (SD) & Value (SD) & $\bar{x}(\mathrm{SD})$ & Min & Max & $\mathrm{SD}^{2}$ \\
\hline $\mathrm{RI}$ & $0.57( \pm 0.04)$ & $0.57( \pm 0.04)$ & $0.57( \pm 0.06)$ & $0.59( \pm 0.04)$ & $0.6( \pm 0.04)$ & $0.58( \pm 0.014)$ & 0.57 & 0.6 & 0.0002 \\
\hline $\mathrm{PI}$ & $1.21( \pm 0.51)$ & $1.22( \pm 0.19)$ & $1.25( \pm 0.44)$ & $1.25( \pm 0.8)$ & $1.3( \pm 0.11)$ & $1.24( \pm 0.035)$ & 1.21 & 1.3 & 0.00123 \\
\hline $\mathrm{S} / \mathrm{D}$ & $2.2( \pm 0.31)$ & $2.24( \pm 0.34)$ & $2.22( \pm 0.28)$ & $2.3( \pm 0.3)$ & $2.49( \pm 0.36)$ & $2.29( \pm 0.117)$ & 2.2 & 2.2 & 0.0139 \\
\hline \multicolumn{7}{c}{ Time (min.) } & 45 & 60 & \\
\hline
\end{tabular}

RI - (resistance index, Pourcelot index), PI - (pulsatility index, Gosling's index), S/D - (systolic/diastolic index)

Table 3. Values of Pearson correlation coefficients $(r)$ and Spearman correlation coefficients ( $r h o$ )

\begin{tabular}{lll}
\hline & $r$ & $r h o$ \\
\hline RI \& PI $(\mathrm{p}<0.001)$ & 0.857 & 0.739 \\
\hline RI \& S/D $(\mathrm{p}<0.05)$ & 0.930 & 0.941 \\
\hline PI \& S/D $(\mathrm{p}<0.001)$ & 0.913 & 0.754 \\
\hline
\end{tabular}

RI - (resistance index, Pourcelot index), PI - (pulsatility index, Gosling's index), S/D - (systolic/diastolic index) 


\section{Discussion}

The pig is a recognised model of research in many branches of medicine. For this reason, the creation of a rich morphometric data library of this species is expected to facilitate the design of scientific research. This assumption also applies to data on kidney vascularity, a sensitive element of intra-body homeostasis $(17,26)$. Doppler tests are a recognised technique for testing blood flows and in the case of humans are conducted without the use of premedication, which may cause false results. It should be noted that the pig is a difficult patient for ultrasound examinations, and testing without the use of pharmacological agents is biased and even impossible to perform. Therefore, during experimental studies it is essential to establish an appropriate anaesthetic protocol to maintain proper blood flow and to avoid errors and falsifications of the perfusion measurement. Many researchers are mindful of this problem while researching other animal species. The combination of atropine, diazepam, acepromazine, and ketamine reduces RI values in healthy dogs (22) but it does not affect this value in cats (23). Anaesthesia with isofluorane increases the value of RI and PI in the cat (16). Some authors presented the problem of estimating the RI in the kidneys of healthy dogs. A physiological difference between the RI of the left and right renal artery in Persian cats was presented by Carvalho and Chammas (3). Similar results were observed during clinical work in dogs and cats $(9,24)$. Chu et al. (4) presented findings concerning the value of the RI in the rabbit renal artery, discovering no adverse effects following the administration of amobarbital sodium during anaesthesia. In research on rabbits, propofol had minimal effect on haemodynamics, whereas combinations of xylazine-ketamine and thiopental resulted in a significant decrease in RI value (10). The propofol studies performed by Gaviria et al. (6) showed its limited effect on haemodynamic parameters.

The anaesthetic protocol was devised on the basis of personal experience and literature reports and allowed a quick, undistorted, and practical examination. The anaesthesia time may cause changes in haemodynamic parameters, and continued examination including prolonged pressure on the kidney may cause a reduction in the late-diastolic velocity and an increase in the RI (32). The maintenance of blood flow in human and animal kidneys is a frequently discussed topic. The pig is a better animal model than rodents in many areas of biomedical research $(13,31)$. The research carried out on the pig model mainly investigated the change of the $\mathrm{RI}$ in the interlobar kidney artery (21) or experimentally induced pathological supply to the arterial kidney (20). No literature describes changes in RI, PI, or S/D during blood flow in the normal segmental artery of the pig kidney (20).
The kidney segmental artery is an interlobar artery readily accessible to the duplex Doppler and early flow disturbances in the arterial vascular system are easy to notice (33). In our research, we demonstrated a constant, one-way blood flow in the diastolic phase in the segmental artery of the swine kidney. This is a feature of low-resistance blood flow, typical in vessels supplying parenchymal organs (Fig. 1). The angle of the spectrum ascending arm depends on the heart systole which accelerates the ejected blood volume. The angle of descent depends on the size and velocity of blood flow in the late-diastolic period and end-diastolic volume. That characterises the peripheral resistance. We proved that the flow spectrum in the segmental kidney artery has lower PSV and PSV/LDV ratio. Moreover, it was also noticed in the filling of the spectral window, comparing the renal to an interlobar artery. There is a visible reduction of the PSV to LDV ratio (flattening of the graph), spectral window filling, and a decrease of PSV value (when comparing segmental to renal arteries) (Figs 1 and 2). The determined low values of variance $\left(\mathrm{SD}^{2}\right)$ indicated coherence and a slight dispersion of the obtained results (Table 3). The flow spectrum and shape of the graph curve depend on variables such as systolic heart capacity, vascular size, elasticity of the vessel walls, flow character, and peripheral resistance; these were shown as a visual presentation on a spectrogram. The RI values, $\bar{x}$ RI, and the value of variance indicate that the spread of the obtained values in the data set was faint. The minimum and maximum PI values, $\bar{x}$ PI and the low SD value similarly confirm the consistency of the obtained results. Also the determined value of variance $\left(\mathrm{SD}^{2}\right)$ indicates a slight dispersion of values in the set. In the case of the $\mathrm{S} / \mathrm{D}$, their narrow range and the $\bar{x} \mathrm{~S} / \mathrm{D}$, similarly to the previous index values, confirm the consistency of results again; the value of variance $\left(\mathrm{SD}^{2}\right)$ shows low dispersion of values in the data set (Table 2). Double analysis of the relationship between changes in the value of individual indices showed a high and almost certain positive correlation confirmed by high correlation coefficients $r$ (Pearson) and rho (Spearman) (Table 3). The values of parameters and indices determined during the research (Tables 1 and 3 ) indicate their similarity to the values established for humans, including children $(28,29,30$, 31). This observation may be important in the planning of transplant studies and research on human kidney pathologies using the pig as an appropriate animal model. The pig model seems to be more popular for this kind of research due to societal pressure to reduce research on monkeys and dogs. Objectivisation of research in the form of numerical data is a beneficial and convenient form of result optimisation. It should be emphasised, however, that particularly in clinical conditions the interpretation of index values without reference to the image of the spectrum or pulse and general pressure values does not allow an unambiguous assessment of the haemodynamic situation to be made 
(34) and should be carried out with strict regard to the general condition of the patient (28).

In conclusion, the swine blood volume is approximately $70-80 \mathrm{~mL} / \mathrm{kg}$ and is similar to the human blood volume $(60-98 \mathrm{~mL} / \mathrm{kg})$. Flow rates up to $60 \mathrm{~cm} / \mathrm{sec}$ in a pig (maximum systolic velocity) correspond to flows in the non-stenotic arteries of the human kidney $(17,26)$. PSV and LDV parameters, and RI, PI, and S/D indices (Tables 1 and 2) indicate similarity to the values empirically established for humans $(1,2,7,11,14,19,35)$. This is important for the planning of transplant studies and research on human kidney pathologies using the pig model.

Conflict of Interests Statements: The authors declare that there is no conflict of interests regarding the publication of this article.

Financial Disclosure Statement: The authors declare that this study was financed by the statutory activity of the Silesian Veterinary Polyclinic Association and the authors' own funds.

Animal Rights Statement: The authors declare that the experiments on animals were conducted in accordance with local Ethical Committee laws and regulations as regards care and use of laboratory animals. The consent for the research was granted by the First Local Ethics Committee for Animal Experiments of the Jagiellonian University in Kraków.

\section{References}

1. Atalabi O.M., Afolabi O.S., Asinobi A.O.: Renal Doppler indices in children with nephrotic syndrome: findings from a tertiary hospital in Nigeria. Niger. J Physiol Sci 2015, 30, $17-23$.

2. Bude R.O., DiPietro M.A., Platt J.F., Rubin J.M., Miesowicz S., Lundquist C.: Age dependency of the renal resistive index in healthy children. Radiology 1992, 184, 469-473.

3. Carvalho C.F., Chammas M.C.: Normal Doppler velocimetry of renal vasculature in Persian cats. J Feline Med Surg 2011, 13, 399-404.

4. Chu Y., Liu H., Xing P., Lou G., Wu C.: The morphology and haemodynamics of the rabbit renal artery: evaluation by conventional and contrast-enhanced ultrasonography. Lab Anim 2011, 45, 204-208.

5. Commission of the European Communities. Third report from the Commission to the Council and the European Parliament on the statistics on the number of animals used for experimental and other scientific purposes in the member states of the European Union. Brussels, 2002.

6. Gaviria E.J., Restrepo J.G., Marín J.D., Arango G., Aramburo D., Franco F., Tintinago L.F.: Evaluation of propofol as an anesthetic in swine tracheal transplant surgery. Rev Col Cienc Pec 2007, 20, 447-454.

7. Gudmundsson S., Maršál K.: Doppler ultrasound examination of the renal artery in healthy women, normotensive pregnant women, and in pre-eclampsia. Ultrasound Obstet Gynecol 1991, $1,258-260$.

8. Henes F.O., Regier M., Bannas P., Henker M., Heinemann A., Sperhake J., Begemann P.G., Adam G., Groth M.: Early timerelated course of image findings in postmortem MRI: typical findings and observer agreement in a porcine model. Leg Med 2017, 28, 15-21.

9. In-Chul P., Hye-Sun L., Jong-Taek K., So-Jeong N., Ran Ch., Ki-Seok O., Chang-Ho S., Changbaig H.: Ultrasonographic evaluation of renal dimension and resistive index in clinically healthy Korean domestic short-hair cats. J Vet Sci 2008, 9, 415-419.

10. Kaya M., Pekcan Z., Sen Y., Boztok B., Senel O.O., Bumin A.: Effects of short-acting anaesthetics on haemodynamic function as determined by Doppler US in rabbits. Kafkas Univ Vet Fak Derg 2011, 17, 713-719.

11. Keogan M., Kliewer M., Hertzberg B., DeLong D.M., Tupler R.H., Carroll B.A.: Renal resistive indexes: variability in Doppler US measurement in a healthy population. Radiology 1996, 199, 165-169.

12. Kim S.H.: The usefulness of pulsatile flow detection in measuring resistive index in renal Doppler US. Korean J Radiol 2002, 3, 45-48.

13. Kujawa-Szewieczek A., Kolonko A., Chudek J., Więcek A.: No effect of pretransplantation bilateral nephrectomy on the resistive indices measured in the kidney allograft. Transplant Proc 2014, $46,2602-2605$.

14. Lin G.J., Cher T.W.: Renal vascular resistance in normal children - a color Doppler study. Pediatric Nephrol 1997, 11, $182-185$.

15. Lind N.M., Moustgaard A., Jelsing J., Vajta G., Cumming P., Hansen A.K.: The use of pigs in neuroscience - modeling brain disorders. Neurosci Biobehav Rev 2007, 31, 728-743.

16. Mitchell S.K., Toal R.L., Daniel G.B., Rohrbach B.W.: Evaluation of renal hemodynamics in awake and isofluoraneanesthetized cats with pulsed-wave Doppler and quantitative renal scintigraphy. Vet Radiol Ultrasound 1998, 39, 451-458.

17. Möckel M., Scheinert D., Potapov E.V., Wellnhofer E., Combé V., Nasseri B.A., Maier D., Meyer S., Yankah C., Hetzer R., Frei U., Eckardt K.U.: Continuous measurements of renal perfusion in pigs by means of intravascular Doppler. Kidney Int 2001, 59, 1439-1447.

18. Naqvi J., Yap K.H., Ahmad G., Ghosh J.: Transcranial Doppler ultrasound: a review of the physical principles and major applications in critical care. Int J Vasc Med 2013, 6, 1-13.

19. Ozel D., Ozel B.D., Ozkan F., Kutlu R.: Evalution of renal involvement in patients with Behçet disease: Need to be aware about potential hypertension in long term follow up? Pol J Radiol 2016, 81, 5-9.

20. Pope J.C., Hernanz-Schulman M., Showalter P.R., Cole T.C., Schrum F.F., Szurkus D., Brock J.W.: The value of Doppler resistive index and peak systolic velocity in the evaluation of porcine renal obstruction. J Urol 1996, 156, 730-733.

21. Rawashdeh Y.F., Mortensen J., Horlyck A., Olsen K.O., Fisker R.V., Schroll L., Frokiaer J.: Resistive index: an experimental study of normal range in the pig. Scand J Urol Nephrol 2000, 34, 10-14

22. Rivers B.J., Walter P.A., Letourneau J.G., Finlay D.E., Ritenour E.R., King V.L., O’Brien T.D., Polzin D.J.: Duplex Doppler estimation of resistive index in arcuate arteries of sedated, normal female dogs: implications for use in the diagnosis of renal failure. J Am Anim Hosp Assoc 1997, 33, 69-76.

23. Rivers B.J., Walter P.A., O'Brien T.D.: Duplex Doppler estimation of Pourcelot resistive index in arcuate arteries of sedated normal cats. J Vet Intern Med 1996, 10, 28-33.

24. Rivers B.J., Walter P.A., Polzin D.J., King V.L.: Duplex doppler estimation of intrarenal pourcelot resistive index in dogs and cats with renal disease. J Vet Intern Med 1997, 11, 250-260.

25. Rodrigues A.C., Santos L.C.D., Otsuki D.A., Saad K.R., Saad P.F., Montero E.F.S., Utiyama E.M.: Animal model of continuous peritoneal lavage with vacuum peritoneostomy. Acta Cir Bras 2017, 32, 467-474.

26. Sauerbrey A., Hindel S., Maaß M., Krüger Ch., Wissmann A., Kramer M., Nafz B., Lüdemann L.: Establishment of a swine model for validation of perfusion measurement by dynamic 
contrast-enhanced magnetic resonance imaging. BioMed Res Int 2014, 7, 1-9.

27. Shoemarker W.: Relation of oxygen transport patterns to the pathophysiology and therapy of shock states. Intensive Care Med 1987, 13, 230-243.

28. Shokeir A.A., Abdulmaaboud M.: Prospective comparison of non-enhanced helical computerized tomography and Doppler US for the diagnosis of renal colic. J Urol 2001, 165, 1082-1084.

29. Stygar A.H., Dolecheck K.A., Kristensen A.R.: Analyses of body weight patterns in growing pigs: a new view on body weight in pigs for frequent monitoring. Animal 2018, 12, 295-302.

30. Sullivan T.P., Eaglstein W.H., Davis S.C., Mertz P.: The pig as a model for human wound healing. Wound Repair Regener 2001, $9,66-76$.
31. Swindle M.M., Makin A., Herron A.J., Clubb F.J., Frazier K.S.: Swine as models in biomedical research and toxicology testing. Vet Pathol 2012, 49, 344-356.

32. Takano R., Ando Y., Taniguchi N., Itoh K., Asano Y.: Power Doppler sonography of the kidney: effect of Valsalva's maneuver. J Clin Ultrasound. 2001, 29, 384-388.

33. Trafidło T., Gaszyński W., Gaszyński T.: The contemporary conversion of the haemodynamic monitoring paradigms in a shock. Anestezjol Ratownictwo 2010, 4, 99-110.

34. Tublin M.E., Bude R.O., Platt J.F.: The resistive index in renal Doppler sonography: where do we stand? Am J Roentgenol 2003, 180, 885-892.

35. Zhang H., Xing X., Wang Z., He M.: Evaluation of split renal function for children with kidney diseases by renal and vascular color ultrasonography. Ultrasound Med Biol 2018, 44, 2602-2608. 